\title{
Bariatric Revisionary Surgery for Failed or Complicated Vertical Banded Gastroplasty (VBG): Comparison of VBG Reoperation (re-VBG) versus Roux-en-Y Gastric Bypass-on-VBG (RYGB-on-VBG)
}

\author{
Stefano Cariani, Laura Agostinelli, Luca Leuratti, Eleonora Giorgini, \\ Pietro Biondi, and Enrico Amenta \\ Department of Emergency/Urgency, General Surgery and Transplantation, University of Bologna, 40138 Bologna, Italy
}

Correspondence should be addressed to Stefano Cariani, stefano.cariani@unibo.it

Received 2 July 2009; Accepted 14 September 2009

Academic Editor: Pietro Forestieri

Copyright (C) 2010 Stefano Cariani et al. This is an open access article distributed under the Creative Commons Attribution License, which permits unrestricted use, distribution, and reproduction in any medium, provided the original work is properly cited.

\begin{abstract}
Background. Revision of failed bariatric procedures is a significant challenge for bariatric surgeons, because of the increasing number of recurring morbid obesity or complications, especially in patients with a previous Vertical Banded Gastroplasty (VBG). Methods. Since November 1998, 109 patients with failed or complicated VBG were followed in a retrospective study. 49 patients underwent re-VBG and, since 2004, 60 underwent Roux-en-Y Gastric Bypass-on-Vertical Banded Gastroplasty (RYGB-on-VBG). Results. At 3 years follow-up, mean BMI decreased from 37.4 to $31.2 \mathrm{Kg} / \mathrm{m}^{2}$ in the first group, and from 35.0 to $28.4 \mathrm{Kg} / \mathrm{m}^{2}$ in the second. Early complications were $7(14.3 \%)$ in the first group and $4(6.5 \%)$ in the second; late complications were $33(59.1 \%)$ and $11(18.3 \%)$, respectively. Conclusion. Although both operations seem to be effective as bariatric revision procedures in terms of BMI, the mid-term outcomes of RYGB-on-VBG demonstrate the lowest rate of complications and better quality of life.
\end{abstract}

\section{Introduction}

The high prevalence of morbid obesity in the world has resulted in many countries performing bariatric surgery, which has proven to be more effective for long-term weight loss than integrated nonsurgical intervention.

Over the past 20 years, thousands of patients have undergone gastric-restrictive procedures, usually vertical banded gastroplasty (VBG).

The VBG procedure is no longer performed in the US, although there are a small number of patients who still receive this procedure worldwide, and the VBG has been largely supplanted by the Roux-en-Y Gastric Bypass (RYGB). On the other hand, it is critical to achieve good outcomes in patients who have undergone VBG and who have fully comprehended that, following bariatric surgery, careful dietary restriction is still required. Patients who do not comply with postoperative instructions represent the main reason why there are so many failures of VBG over the long term.

Since the 1980s, it has been reported that some patients may require a second surgical intervention due to the presence of complications or insufficient weight loss after VBG; however, over time, the number of patients who need revisionary bariatric surgery has grown.

It is increasingly likely that the bariatric surgeon will be confronted with a dilemma regarding how to treat the patient who is experiencing recurring morbid obesity and/or complications after a restrictive procedure.

The aim of the revisionary procedure is to treat complications and to achieve further weight loss in patients with unsuccessful weight reduction when it is ascertained that nonsurgical management is not working.

Revision of failed bariatric procedures is a significant challenge for every bariatric surgeon, especially when the previous surgery was a gastric restrictive procedure [1-9] like 
the VBG, where the first difficulty is to separate the liver from the anterior gastric wall, potentially leading to tissue ischemia and leaks.

Many authors have reported a high rate of complications after revisionary bariatric surgery [5-7, 10-16], while others have reported a low rate of complications [9, 17-21]. After weighing the advantages and disadvantages of a second bariatric procedure, in comparison to the presence of disease, the balance seems to favor reoperation.

No specific reoperative bariatric surgical standard exists for the selection of a procedure; the vast majority of bariatric surgeons perform revisionary surgery on the basis of their experience [3-9, 17, 20, 21].

Selecting a particular type of surgical treatment must consider the weight loss results after the primary operation, the patient's quality of life, psychological status, general wellbeing, the technique used in the primary operation, the cause of failure, and the extent of the surgeon's experience.

After a VBG failure, in choosing the approach to revisionary surgery, the most common surgical strategies for many surgeons are the following: restoration of a new purely restrictive procedure (re-VBG), conversion to a Roux-en-Y gastric bypass (RYGB), and conversion to a biliopancreatic diversion (BPD).

Usually, if the complication does not affect the patient's weight loss or quality of life, or if the patient had good weight loss until the onset of complications, a second performance of the same restrictive operation should be considered. If the procedure fails because the patient proves to be noncompliant and complications are caused by poor dietary habits, the conversion to a different bariatric procedure to control weight loss is strictly recommended.

The aim of this retrospective study was to evaluate results and complications of two different procedures adopted as bariatric revisionary surgery on patients with failed and complicated VBG.

We have compared the outcomes of restored vertical banded gastroplasty (re-VBG) and conversion to Roux-en $\mathrm{Y}$ gastric bypass on vertical banded gastroplasty (RYGBon-VBG), both performed in open surgery. The RYGB-onVBG $[15,22,23]$ is a modification of the traditional RYGB, developed in 2002 to allow endoscopic and radiological investigation of the excluded stomach.

\section{Methods}

The medical records and follow-up data for all patients, who underwent revisionary bariatric surgery at the hands of our surgical group from January 1998 to December 2008, were reviewed. Demographic characteristics, preoperative conditions, surgical details, operative time, mean hospitalization, morbidity, mortality, weight loss up to 3 years, and complications were analyzed.

In our institution the revisionary bariatric surgery was performed on 146 patients; 134 of these for VBG failures or complications, while the other 12 addressed failures of adjustable gastric banding. Of 134 patients undergoing revisionary surgery for open-VBG complications or failures, 109 were included in a retrospective study. For this study, the
TABLE 1: Revisionary surgery for failed or complicated VBGs: patient characteristics.

\begin{tabular}{|c|c|c|c|}
\hline \multirow{2}{*}{ Characteristic } & \multicolumn{3}{|c|}{ Technique } \\
\hline & Re-VBG & Conversion to RYGB-on-VBG & TOT \\
\hline n. Patients & 49 & 60 & 109 \\
\hline Mean AGE yrs & 46 & 48 & 47 \\
\hline Mean BMI & $37.4 \pm 7.1$ & $35 \pm 8.9$ & $36.1 \pm 7.7$ \\
\hline
\end{tabular}

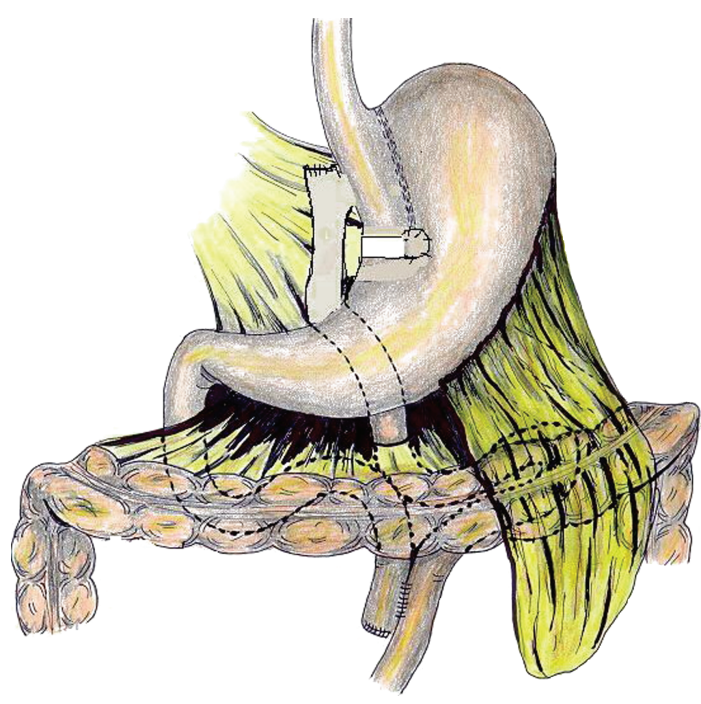

FIGURE 1: Example sketch of a RYGB-on-VBG.

cohort was divided into two homogeneous groups of 49 and 60 patients, undergoing re-VBG and, since 2004, RYGB-onVBG conversion, respectively. Demographic characteristics are shown in Table 1.

The other 25 patients were not included in the study because they underwent different procedures, like traditional RYGB or gastric restoration (i.e., a return to the normal anatomy).

The Roux-en-Y gastric bypass on vertical banded gastroplasty technique differs from the traditional gastric bypass by leaving a small communication (one centimeter of the inside diameter), as in the VBG, between the gastric pouch and the gastric remnant, avoiding definitive gastric exclusion (Figure 1).

To recreate Mason's VBG, we simply restapled the stomach. In cases of pouch dilation or a small staple line disruption, a complementary gastro-gastric anastomosis was performed in order to avoid mucocele of the blind gastric tract.

To perform a RYGB-on-VBG, in cases of staple line disruption or pouch dilation, the first step was to recreate a Mason's VBG. When the gastro-gastric outlet was completely obstructed, we removed the band of the previously performed VBG, to prevent a band erosion. Next, a two-layer hand-sewn gastrojejunostomy creating a $150 \mathrm{~cm}$ retrocolic and antegastric Roux limb, and a side-to-side jejunojejunostomy with a $30 \mathrm{~cm}$ biliopancreatic limb completed the 
TABLE 2: Revisionary surgery for failed or complicated VBGs: causes of reoperation.

\begin{tabular}{lcccc}
\hline Cause & Re-VBG & $\begin{array}{c}\text { Technique } \\
\text { Conversion to } \\
\text { RYGB-on-VBG }\end{array}$ & Total & $\%$ \\
\hline $\begin{array}{l}\text { Weight regain \& } \\
\text { staple line disruption } \\
\begin{array}{l}\text { Vomiting \& stoma } \\
\text { stenosis }\end{array}\end{array}$ & 37 & 35 & 72 & 66 \\
$\begin{array}{l}\text { Weight regain \& } \\
\text { pouch dilation }\end{array}$ & 9 & 11 & 13 & 12 \\
$\begin{array}{l}\text { Vomiting \& kinked } \\
\text { stoma }\end{array}$ & 1 & 4 & 13 & 12 \\
Weight regain & 0 & 9 & 10 & 9 \\
\hline Total & 49 & 60 & 1 & 1 \\
\hline
\end{tabular}

gastric bypass. An abdominal drainage tube was routinely left in place.

All the patients received antibiotic prophylaxis, pharmacological and mechanical antithrombotic prophylaxis.

Postoperatively, all the patients underwent a watersoluble swallow after 72 hours; if a leak was excluded, the abdominal drainage was removed and a liquid diet was initiated. The diet was gradually changed to solid food over the course of one month. All patients were treated with PPIs and prokinetic agents and continued the therapy for two months.

For all patients, follow-up was scheduled at six and twelve months, and annually thereafter, following this procedure for each patient:

(i) clinical control,

(ii) X-ray study with barium,

(iii) upper endoscopy when necessary.

At reoperation, the overall mean age was 47 and the mean BMI was $36.1 \pm 7.7 \mathrm{Kg} / \mathrm{m}^{2}$ (Table 1 ). In the 109 patients with previous VBG, the indications for revision were weight regain with staple-line disruption (n. 72, 66\%) or pouch dilation (n. 13,12\%). We also included cases with no weight regain, with recurrent vomiting due to stenosis of the banded stoma (n. 13,12\%) or kinked stoma (n. 10, 9\%), as well as cases with weight regain with normal surgical anatomy (n. 1, 1\%) (Table 2). We considered the vomiting to be recurrent when it occurred more than two times/week.

To compare the two operations, for each group of patients who we followed for three years following surgery, we focused on the changes in BMI, and on the early and late specific complications. We have not considered the woundrelated complications, because all the procedures were performed in open surgery. The outcomes were compared statistically after obtaining patient consent and IRB approval.

\section{Results}

3.1. First Group: re-VBG. The average operative time was $143 \pm 34$ minutes and the mean hospitalization was $7 \pm 2$ days. For 49 patients, clinical follow-up was recorded for up to 3 years. We observed no operative mortality and the 30day operative morbidity (n. 7, 14.3\%) is listed in Table 3. The rate of early specific surgical complications was $8.1 \%$, due to 4 patients who had a leak of vertical sutures in the upper part of the gastric pouch. Two patients healed with abdominal drainage, empty stomach, TPN and PPI therapy. The other two patients were treated surgically where we performed gastro-gastric anastomosis, and they were excluded from the study, because the return to the normal anatomy is ineffective on weight loss.

Among the 47 eligible patients, the overall rate of late complications was $67.3 \%$ (n. 33), with recurrent vomiting being by far the most frequent (Table 4). It should be emphasized that the vomiting drastically affected the quality of life. Despite the absence of surgical complications, 7/19 patients had to be reoperated upon due to recurrent vomiting. For each of those patients, we performed a gastric restoration (i.e., gastro-gastric anastomosis), because they refused conversion to another bariatric procedure. All the patients who underwent gastro-gastric anastomosis were excluded from weight loss/gain statistics (overall rate of $8.2 \%$, n. 7 ).

Thus, the trend of BMI was evaluated for 40 patients. The mean preoperative body mass index decreased from $37.4 \pm$ $7.1 \mathrm{Kg} / \mathrm{m}^{2}$ to $30.5 \pm 4.6,30.9 \pm 4.6$ and $31.2 \pm 5.3 \mathrm{Kg} / \mathrm{m}^{2}$ after 1,2 , and 3 years, respectively (Table 5 ).

We found gastro-esophageal reflux in $14.3 \%$ of patients (n. 7), often associated with recurrent vomiting due to poor eating habits. This symptom usually improves with time and therapy with PPIs and prokinetic agents.

Three cases of gastro-gastric fistula were detected (rate of $6.1 \%$ ) with barium swallows in asymptomatic patients, two and three years postoperatively, but did not invalidate the outcomes with regard to weight.

3.2. Second Group: RYGB-on-VBG. The average operative time was $183 \pm 27$ minutes and the mean hospitalization time was $8 \pm 2$ days. We had no operative mortality or specific surgical complications. The 30-day operative morbidity is listed in table three (Table 3).

For 57 of the 60 patients, the clinical follow-up was recorded for up to 3 years, with a drop-out rate of $5 \%$. In the 57 evaluated patients, the mean preoperative body mass index decreased from $35.0 \pm 8.9 \mathrm{Kg} / \mathrm{m}^{2}$ to $28.6 \pm 4.4,30.2 \pm 8.0$, and $28.4 \pm 4.5 \mathrm{Kg} / \mathrm{m}^{2}$ after 1,2 , and 3 years, respectively (Table 5).

The overall rate of late complications was $18.3 \%$ (n. 11). No patient reported recurring vomiting (Table 4), while the rate of dumping syndrome was $6.5 \%$ (n. 4). However, dumping syndrome had a tendency to disappear with the passing of time.

We also found heartburn and regurgitation symptoms in $5 \%$ of the patients (n. 3). All of these patients were smokers and with poor eating habits; with PPI and prokinetic agent therapy, they stopped smoking and healed completely.

Three asymptomatic patients developed gastro-gastric fistulae (rate of 5\%) without effects on weight loss. 
TABLE 3: Revisionary surgery for failed or complicated VBGs: 30-day operative and late complication rate.

\begin{tabular}{|c|c|c|c|c|c|c|c|}
\hline \multirow{3}{*}{ Complications } & \multicolumn{7}{|c|}{ Technique } \\
\hline & & \multicolumn{2}{|c|}{ Re-VBG } & \multicolumn{2}{|c|}{ Conversion to RYGB-on-VBG } & \multirow{2}{*}{ TOT } & \multirow{2}{*}{$\%$} \\
\hline & & n. & $\%$ & n. & $\%$ & & \\
\hline \multirow{2}{*}{$\begin{array}{l}\text { 30-days Operative } \\
\text { complications }\end{array}$} & Surgical & 4 & 8.1 & 0 & 0 & 4 & 6.7 \\
\hline & Not-surgical & 3 & 6.1 & 4 & 6.5 & 7 & 6.4 \\
\hline \multicolumn{2}{|c|}{ Late complications } & 33 & 67.3 & 11 & 18.3 & 44 & 40.3 \\
\hline
\end{tabular}

TABLE 4: Outcomes of revisionary surgery for failed or complicated VBGs: long-term complications.

\begin{tabular}{|c|c|c|c|c|c|c|}
\hline \multirow{3}{*}{ Complications } & \multicolumn{6}{|c|}{ Technique } \\
\hline & \multicolumn{2}{|c|}{ Re-VBG } & \multicolumn{2}{|c|}{ Conversion to RYGB-on-VBG } & \multirow{2}{*}{ TOT } & \multirow{2}{*}{$\%$} \\
\hline & n. & $\%$ & n. & $\%$ & & \\
\hline Recurrent Vomiting & 19 & 38.7 & & 0 & 19 & 17.4 \\
\hline Gastro-esophageal reflux & 7 & 14.3 & & 0 & 7 & 6.4 \\
\hline Heartburn and regurgitation & & 0 & 3 & 5 & 3 & 2.8 \\
\hline Acute gastritis & & 0 & 1 & 1.6 & 1 & 0.9 \\
\hline Dumping Syndrome & & 0 & 4 & 6.5 & 4 & 3.7 \\
\hline Gastro-gastric fistula & 3 & 6.1 & 3 & 5 & 6 & 5.5 \\
\hline Outlet sub-stenosis & 1 & 2 & & 0 & 3 & 2.7 \\
\hline Reoperation (gastro-gastric anastomosis) & 7 & 14.3 & & 0 & 7 & 6.4 \\
\hline Total & 33 & 67.3 & 11 & 18.3 & 50 & 45.8 \\
\hline
\end{tabular}

TABLE 5: Outcomes of revisionary surgery for failed or complicated VBGs: changes in BMI.

\begin{tabular}{lcc}
\hline Follow-up & \multicolumn{2}{c}{$\begin{array}{c}\text { Technique } \\
\text { Conversion to RYGBP } \\
\text { on VBG n. 60 }\end{array}$} \\
\hline Operative & $37.4 \pm 7.1$ & $35.0 \pm 8.9$ \\
1 Yr & $30.5 \pm 4.6$ & $28.6 \pm 4.4$ \\
2 Yrs & $30.9 \pm 4.6$ & $30.2 \pm 8.0$ \\
3 Yrs & $31.2 \pm 5.3$ & $28.4 \pm 4.5$ \\
Lost to follow up/\% & $9 / 8.2 \%$ & $3 / 5 \%$ \\
\hline
\end{tabular}

The fistulae were detected during the X-ray exam, one and two years postoperatively.

3.3. First Group versus Second Group. We analyzed the results for mean weight loss and complications in each group, comparing the BMI at 1, 2, and 3 years of follow-up.

To compare the BMI, we have used the "one-way variance test (F-test)", in order to characterize differences between the two groups and within each group of patients. Then using the Student's $t$-test, we compared the results of the two groups (Table 6).

Weight loss appeared to be greater in the second group; however, the difference between the groups was not statistically significant. In order to compare the complications of each group, we applied the chi-square test (Table 7).

The test did not show a significant difference between the two groups of patients with regard to early complications. It showed a very significant difference $(P$ value $<.001)$
TABLE 6: Outcomes of revisionary surgery for failed or complicated VBGs: statistical study to compare changes in BMI between 2 groups.

\begin{tabular}{lccc}
\hline \multirow{2}{*}{ Statistics } & \multicolumn{3}{c}{ Follow-up } \\
& 1 yr & 2 yrs & 3 yrs \\
\hline Variance between the groups $\left(\mathrm{S}^{2}{ }_{\text {bet }}\right)$ & 80.07 & 8.56 & 82.8 \\
Variance into the groups $\left(\mathrm{S}^{2}{ }_{\text {into }}\right)$ & 20.31 & 38.05 & 26.2 \\
$F$ & 3.94 & 0.63 & 3.16 \\
Student $t$-test & 1.98 & 0.47 & 1.18 \\
$P$ & $<.05$ & .63 & .08 \\
\hline
\end{tabular}

TABLE 7: Outcomes of revisionary surgery for failed or complicated VBGs: chi-square test applied to compare 30-day operative and long-term complications.

\begin{tabular}{lcccc}
\hline & \multicolumn{3}{c}{ Technique } \\
Complications & Re-VBG & RYGB-on-VBG & \multicolumn{2}{c}{ Chi Square } \\
& & & $\chi^{2}$ & $P$ \\
\hline 30-days operative & 7 & 4 & 0.988 & .320 \\
Long-term & 29 & 11 & 17.657 & $<.001$ \\
\hline
\end{tabular}

when comparing late complications, demonstrating RYGBon-VBG to be the more effective technique.

\section{Discussion}

In comparison to the primary operation, bariatric surgery revisions are technically more demanding and have a higher risk of postoperative complications. However, revisionary surgery is the best solution for patients who have experienced 


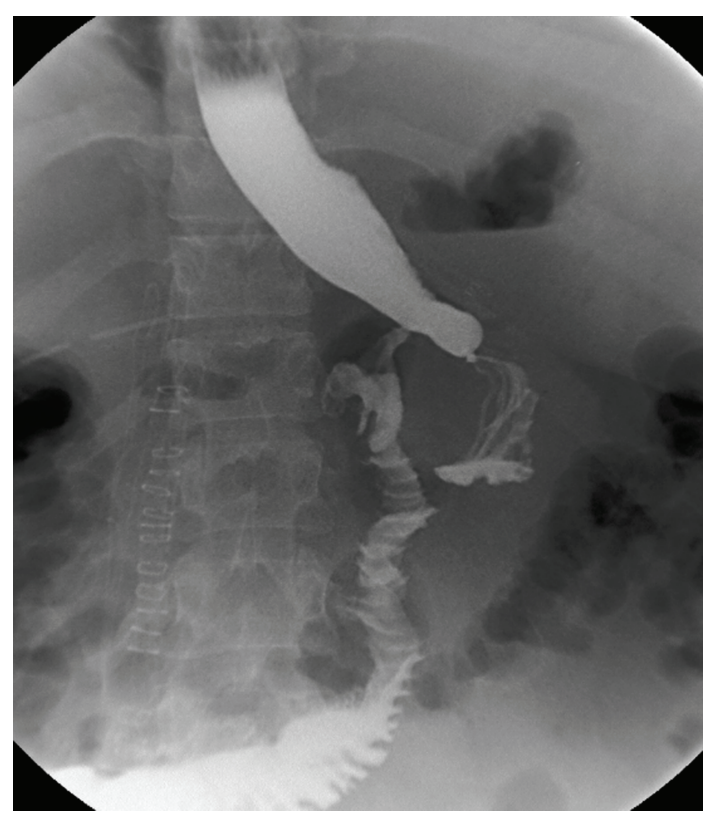

Figure 2: Traditional X-ray studies of a RYGB-on-VBG.

a first bariatric restrictive procedure with poor results or with complications. The aim of a revisionary procedure is to treat complications and to achieve further weight loss in patients with unsuccessful weight reduction or with weight regain.

In this retrospective study on patients undergoing bariatric revisionary surgery, we focused on the management of failures and complications after VBG. The literature reveals a high incidence of revision for this operation, with rates from 10 to $56 \%$.

With regard to the choice of revisionary procedure, several authors have observed that in many cases of re-VBG, further revisionary procedures are required over the longterm. Reports indicate that conversion to an RYGB is more effective than a re-VBG.

At our institution, both operations were adopted: the reVBG from 1998 to 2004 and conversion to the RYGB-onVBG from 2004 to 2008. We performed this modification of standard RYGB because the operation, when adopted as the primary operation $[15,22,23]$, proved to be equivalent to the standard RYGB, while enabling traditional diagnostic evaluation of the bypassed stomach. Moreover, when conversion is needed due to the presence of nausea, dysphagia, and vomiting, stoma stenosis or pouch dilatation, adoption of the RYGB-on-VBG surgical procedure is facilitated, because the gastric pouch does not have to be re-created.

In agreement with many authors, the mid-term outcomes of our patients in terms of weight loss were more effective with the RYGB-on-VBG technique than with the re-VBG; however, the difference between the groups was not statistically significant. Future studies will be necessary to evaluate the positive trend of improved weight loss with RYGB-on-VBG over the long term in more patients.

We observed that in the patients who underwent reVBG, the high incidence of recurrent vomiting drastically and negatively impacted quality of life. Such vomiting was the reason why, in this series, many patients required a revisionary operation.

Even though revisionary bariatric surgery is a challenge due to technical difficulties, we found that the conversion of a failed open VBG into open RYGB-on-VBG was safe and effective. We propose that this technique be used to overcome the issues imposed by an excluded stomach after gastric bypass.

With every patient followed after RYGB-on-VBG, traditional X-ray studies (Figure 2) of the stomach and/or gastroscopy provided a valid examination of the bypassed stomach and duodenum. We believe that it is important to be able to perform these procedures when necessary, because the patients may develop acute complications of the bypassed stomach, duodenum, and biliary tract after gastric surgery, such as hemorrhage and perforation, or cancer.

A traditional barium swallow or an upper endoscopy can be carried out easily and quickly in all hospitals, and in this regard, we believe that the RYGBP-on-VBG improves the safety of the patients. Further investigation will be required to comprehensively elucidate the RYGB-on-VBG operation when performed as revisionary surgery and the long-term effects of this procedure.

\section{References}

[1] S. R. Fox, K. M. Fox, and K. H. Oh, "The gastric bypass for failed bariatric surgical procedures," Obesity Surgery, vol. 6, no. 2, pp. 145-150, 1996.

[2] K. E. Behrns, C. D. Smith, K. A. Kelly, and M. G. Sarr, "Reoperative bariatric surgery: lessons learned to improve patient selection and results," Annals of Surgery, vol. 218, no. 5, pp. 646-653, 1993.

[3] B. Van Wageningen, F. J. Berends, B. Van Ramshorst, and I. F. M. Janssen, "Revision of failed laparoscopic adjustable gastric banding to Roux-en-Y gastric bypass," Obesity Surgery, vol. 16, no. 2, pp. 137-141, 2006.

[4] J. M. Calmes, V. Giusti, and M. Suter, "Reoperative laparoscopic Roux-en-Y gastric bypass: an experience with 49 cases," Obesity Surgery, vol. 15, no. 3, pp. 316-322, 2005.

[5] M. Suter, V. Giusti, E. Héraief, and J.-M. Calmes, "Band erosion after laparoscopic gastric banding: occurrence and results after conversion to Roux-en-Y gastric bypass," Obesity Surgery, vol. 14, no. 3, pp. 381-386, 2004.

[6] A. Iannelli, D. Amato, P. Addeo, et al., "Laparoscopic conversion of vertical banded gastroplasty (Mason MacLean) into Roux-en-Y gastric bypass," Obesity Surgery, vol. 18, no. 1, pp. 43-46, 2008.

[7] P. Mognol, D. Chosidow, and J. P. Marmuse, "Roux-en-Y gastric bypass after failed vertical banded gastroplasty," Obesity Surgery, vol. 17, no. 11, pp. 1431-1434, 2007.

[8] A. A. Gumbs, A. Pomp, and M. Gagner, "Revisional bariatric surgery for inadequate weight loss," Obesity Surgery, vol. 17, no. 9, pp. 1137-1145, 2007.

[9] P. Topart, G. Becouarn, and P. Ritz, "Biliopancreatic diversion with duodenal switch or gastric bypass for failed gastric banding: retrospective study from two institutions with preliminary results," Surgery for Obesity and Related Diseases, vol. 3, no. 5, pp. 521-525, 2007.

[10] R. F. Capella and J. F. Capella, "Converting vertical banded gastroplasty to a lesser curvature gastric bypass: technical 
considerations," Obesity Surgery, vol. 8, no. 2, pp. 218-224, 1998.

[11] W. Vaneerdeweg, G. Hubens, L. Van Gaal, and E. Eyskens, "Operations for failed vertical banded gastroplasty," Acta Chirurgica Belgica, vol. 94, no. 4, pp. 203-206, 1994.

[12] H. J. Sugerman and J. L. Wolper, "Failed gastroplasty for morbid obesity. Revised gastroplasty versus Roux-Y gastric bypass," American Journal of Surgery, vol. 148, no. 3, pp. 331336, 1984.

[13] R. A. Hunter, J. M. K. Watts, R. E. Dunstan, et al., "Revisional surgery for failed gastric restrictive procedures for morbid obesity," Obesity Surgery, vol. 2, no. 3, pp. 245-252, 1992.

[14] J. F. Sweeney, S. E. Goode, and A. S. Rosemurgy, "Redo gastric restriction: a higher risk procedure," Obesity Surgery, vol. 4, no. 3, pp. 244-247, 1994.

[15] S. Cariani and E. Amenta, "Three-year results of Roux-en$\mathrm{Y}$ gastric bypass-on-vertical banded gastroplasty: an effective and safe procedure which enables endoscopy and X-ray study of the stomach and biliary tract," Obesity Surgery, vol. 17, no. 10, pp. 1312-1318, 2007.

[16] S. Cariani, D. Nottola, S. Grani, G. Vittimberga, A. Lucchi, and E. Amenta, "Complications after gastroplasty and gastric bypass as a primary operation and as a reoperation," Obesity Surgery, vol. 11, no. 4, pp. 487-490, 2001.

[17] J. A. Sapala, R. J. Bolar, J. P. Bell, and M. A. Sapala, “Technical strategies for converting the failed vertical banded gastroplasty to the Roux-en-Y gastric bypass," Obesity Surgery, vol. 3, no. 4, pp. 400-409, 1993.

[18] E. Kfoury and A. Vanguri, "Distal Roux-en-Y gastric bypass conversion operation for failed vertical banded gastroplasty," Obesity Surgery, vol. 3, no. 1, pp. 41-43, 1993.

[19] H. J. Sugerman, J. M. Kellum Jr., E. J. DeMaria, and H. D. Reines, "Conversion of failed or complicated vertical banded gastroplasty to gastric bypass in morbid obesity," American Journal of Surgery, vol. 171, no. 2, pp. 263-269, 1996.

[20] W. G. Van Gemert, M. M. Van Wersch, J. W. M. Greve, and P. B. Soeters, "Revisional surgery after failed vertical banded gastroplasty: restoration of vertical banded gastroplasty or conversion to gastric bypass," Obesity Surgery, vol. 8, no. 1, pp. 21-28, 1998.

[21] C. E. Yale, "Conversion surgery for morbid obesity: complications and long-term weight control," Surgery, vol. 106, no. 3, pp. 474-480, 1989.

[22] S. Cariani, P. Palandri, E. Della Valle, et al., "Italian multicentric experience of Roux-en-Y gastric bypass on vertical banded gastroplasty: four-years results of effective and safe innovative procedure enabling traditional endoscopic and radiographic study of bypassed stomach and biliary tract," Surgery for Obesity and Related Diseases, vol. 4, no. 1, pp. 16-25, 2008.

[23] S. Cariani, G. Vittimberga, S. Grani, A. Lucchi, M. Guerra, and E. Amenta, "A functional Roux-en-Y gastric bypass to avoid gastric exclusion: 1-year results," Obesity Surgery, vol. 13, no. 5, pp. 788-791, 2003. 


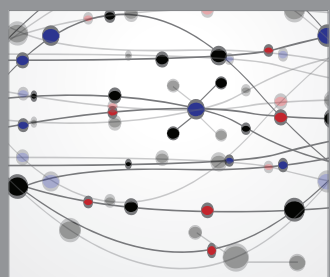

The Scientific World Journal
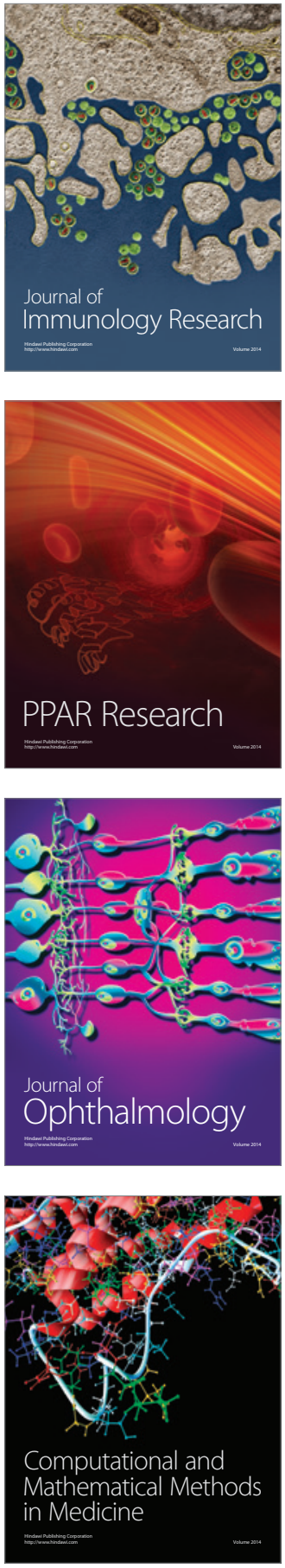

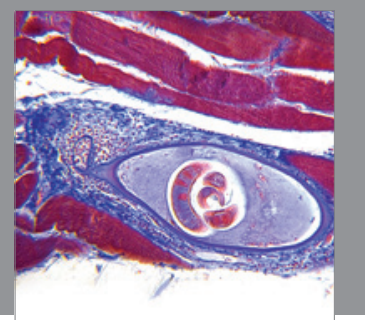

Gastroenterology

Research and Practice
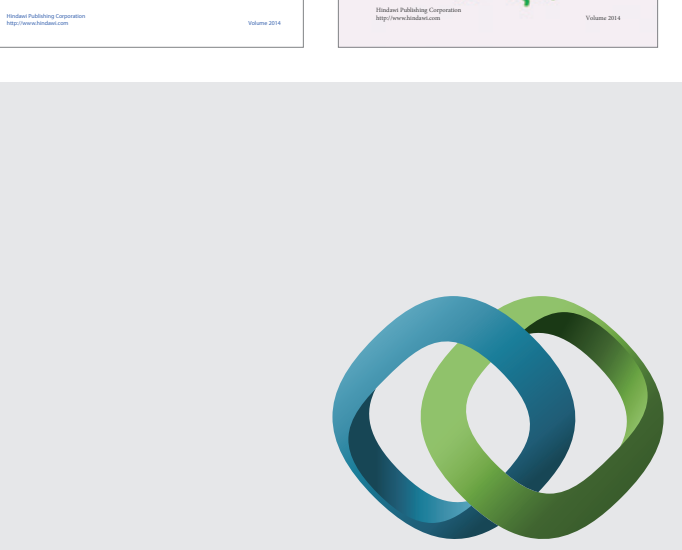

\section{Hindawi}

Submit your manuscripts at

http://www.hindawi.com
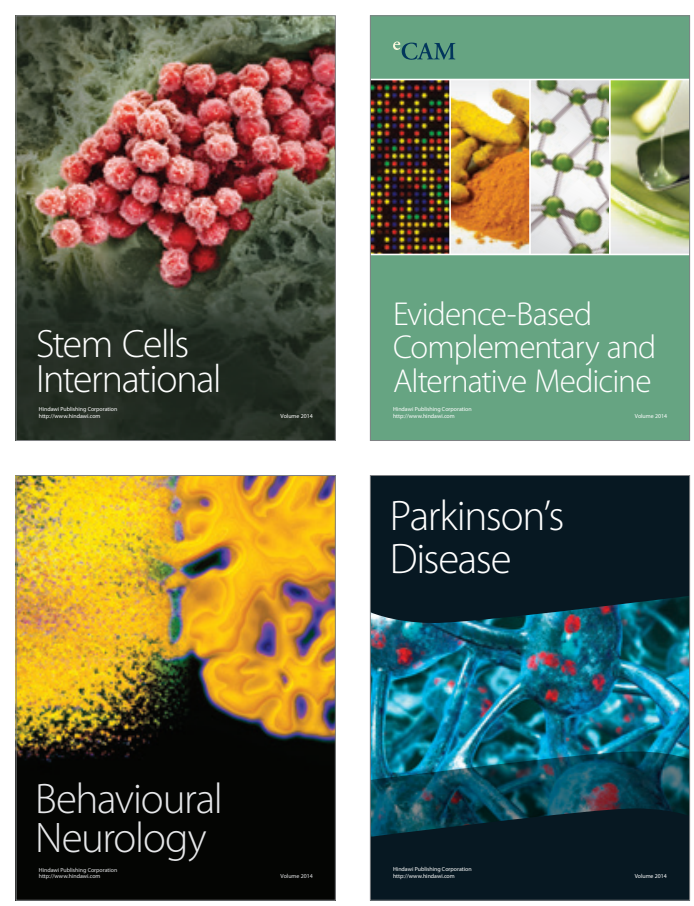

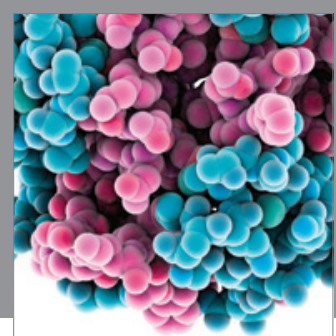

Journal of
Diabetes Research

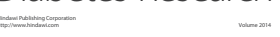

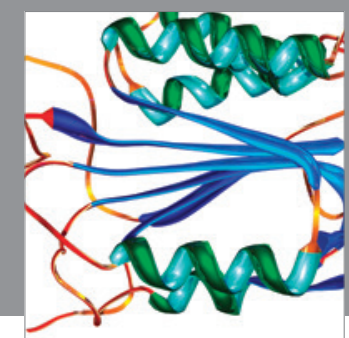

Disease Markers
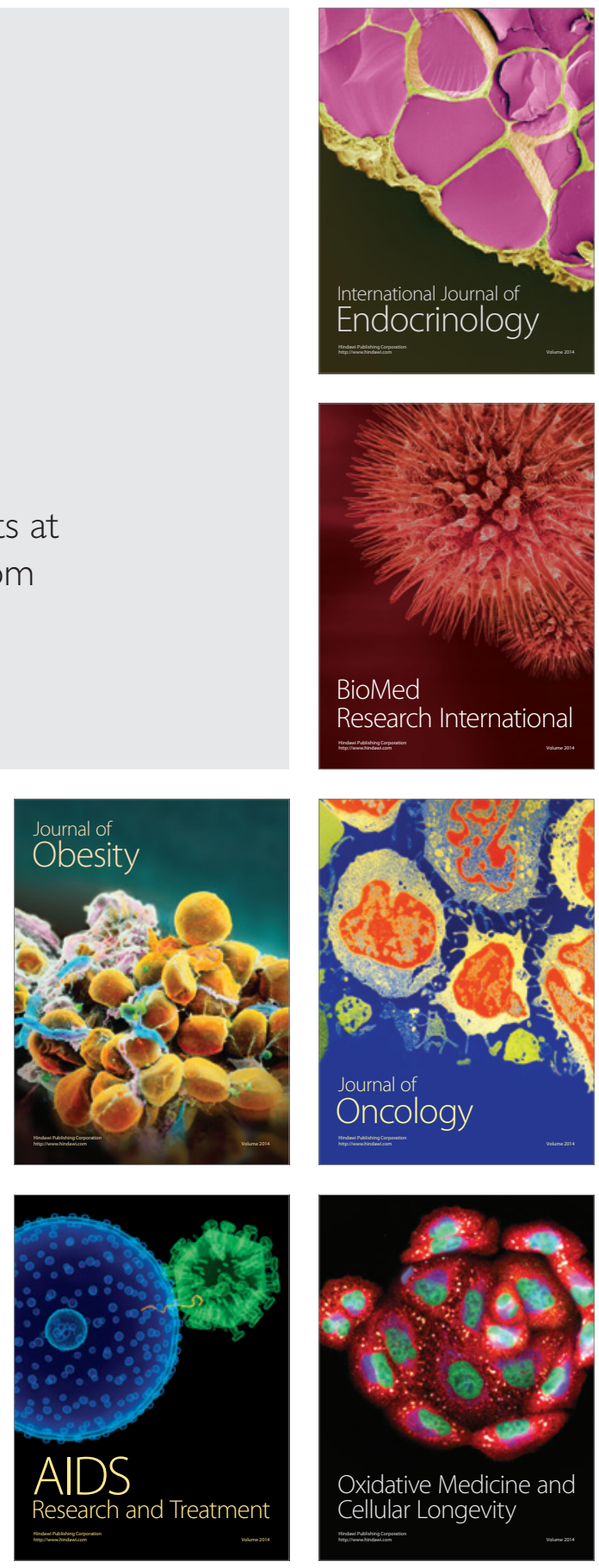